\title{
Obstetric spinal hypotension: Preoperative risk factors and the development of a preliminary risk score - the PRAM score
}

\author{
D G Bishop, ${ }^{1} \mathrm{MB}$ ChB, FCA; C Cairns, ${ }^{1} \mathrm{MB}$ ChB, FCA; M Grobbelaar, ${ }^{1} \mathrm{MB}$ ChB, FCA; R N Rodseth, ${ }^{1,2} \mathrm{MB}$ ChB, FCA, MMed, \\ Cert Crit Care (SA), MSc, PhD \\ ${ }^{1}$ Metropolitan Department of Anaesthetics, Critical Care and Pain Management, School of Clinical Medicine, Nelson R Mandela School of \\ Medicine, College of Health Sciences, University of KwaZulu-Natal, Pietermaritzburg, South Africa \\ ${ }^{2}$ Outcomes Research Consortium, Cleveland Clinic, Ohio, USA
}

Corresponding author: D G Bishop (davidgbishop@gmail.com)

\begin{abstract}
Background. Obstetric spinal hypotension is a common and important problem during caesarean delivery. Identifying patients at risk for hypotension may guide clinical decision-making and allow timeous referral.

Objective. Using preoperative risk factors, to develop a simple scoring system to predict systolic hypotension.

Methods. This prospective, single-centre, observational study of patients undergoing elective or urgent caesarean delivery assessed body mass index, baseline heart rate, baseline mean arterial pressure (MAP), maternal age, urgency of surgery (elective v. non-elective) and preoperative haemoglobin concentration as predictors of spinal hypotension (systolic blood pressure $<90 \mathrm{mmHg}$ ). We used empirical cut-point estimations in a logistic regression model to develop a scoring system for prediction of hypotension.

Results. From 504 eligible patients, preoperative heart rate (odds ratio (OR) 1.02, 95\% confidence interval (CI) 1.00 - 1.03 ; $p=0.012$ ), preoperative MAP (OR 0.97, 95\% CI 0.95 - 0.98; $p<0.001$ ) and maternal age (OR 1.05, 95\% CI $1.02-1.08$; $p=0.002$ ) were found to be predictors of hypotension. We derived a preliminary scoring system (pulse rate $>90 \mathrm{bpm}$, age $>25$ years, $\mathrm{MAP}<90 \mathrm{mmHg}-$ the $\mathrm{PRAM}$ score) for the prediction of systolic hypotension following obstetric spinal anaesthesia. Patients with three factors had a $53 \%$ chance of developing hypotension, compared with the overall incidence of $30 \%$. The PRAM score showed good discrimination, with a $c$-statistic of 0.626 (95\% CI 0.576 - 0.676) and good calibration.

Conclusions. Preoperative heart rate, preoperative MAP and maternal age were predictive of hypotension in elective and emergency caesarean delivery. The PRAM score shows promise as a simple, practical means to identify these patients preoperatively, but requires prospective validation.
\end{abstract}

S Afr Med J 2017;107(12):1127-1131. DOI:10.7196/SAMJ.2017.v107i12.12390

Spinal hypotension during caesarean delivery occurs commonly and, depending on the definition used, may have an incidence as high as $71 \% .^{[1]}$ Hypotension during caesarean delivery is a potentially life-threatening event and is associated with nausea and vomiting, ${ }^{[2]}$ loss of consciousness, cardiac arrest, death and fetal compromise. ${ }^{[3]}$ In South Africa (SA), anaesthesia-related maternal mortality accounts for $2 \%$ of maternal deaths, with over half of these due to spinal hypotension. ${ }^{[4]}$ Identifying patients at risk of developing intraoperative spinal hypotension could enable targeted interventions to reduce mortality. Preoperative risk prediction for intraoperative hypotension has yielded mixed results, with some methods requiring specialised equipment that is not routinely available. ${ }^{[5]}$ There are also no practical scoring systems to better quantify the risk of intraoperative hypotension. Scoring systems could potentially enhance anaesthetic planning, guide clinical decision-making and make timeous referral possible where appropriate.

\section{Objective}

To assess practical preoperative risk factors for the development of obstetric spinal hypotension, with the aim of developing a simple risk scoring system.

\section{Methods \\ Design}

We undertook a prospective, single-centre, observational study of patients undergoing caesarean delivery under spinal anaesthesia in an SA regional hospital.

\section{Ethical considerations}

Ethical approval was given by the University of KwaZulu-Natal Ethics Committee (ref. no. BE309/15), and permission to conduct the study was given by hospital management and the provincial Department of Health (ref. no. 2015RP27_702). Owing to the observational nature of the data collected, the need for written consent from patients was waived by both the ethics board and the health department. Information regarding the study was made available to patients in the form of an information pamphlet and displayed in the labour ward and obstetric theatres.

\section{Setting}

The study was conducted in a regional referral centre in SA. It caters for both routine and complex obstetric cases. The hospital runs two obstetric theatres during normal working hours and one theatre after hours. The service is run largely by junior medical officers with an 
average of 18 months' anaesthesia experience. Consultant anaesthetic supervision is available if required.

\section{Participants}

Recruitment of patients scheduled for caesarean delivery under spinal anaesthesia between $07 \mathrm{~h} 30$ and $16 \mathrm{~h} 00$ on normal working days took place from 29 June 2015 to 25 November 2015. For this analysis we included all patients undergoing spinal anaesthesia, i.e. both elective and emergency cases. Patients who underwent conversion to general anaesthesia during the time period assessed (insertion of spinal until 15 minutes after delivery of the baby) were excluded from analysis.

\section{Management and data collection}

Relevant patient data recorded during the initial assessment included height, weight, preoperative haemoglobin concentration, maternal age and urgency of surgery. Baseline vital signs (heart rate and non-invasive blood pressure) were recorded in theatre in the left lateral position prior to administering spinal anaesthesia. After administration of the spinal, non-invasive blood pressure measurements were made at 1-minute intervals until 15 minutes after delivery. Patients received metoclopramide $10 \mathrm{mg}$ intravenously and sodium citrate $30 \mathrm{~mL}$ per os preoperatively. Spinal anaesthesia was standardised: all patients received $9 \mathrm{mg} 0.5 \%$ hyperbaric bupivacaine with $10 \mu \mathrm{g}$ fentanyl, administered in the sitting position, and were then immediately put into the supine position with a right hip wedge. Hypotension was managed according to unit protocol. Vasopressor boluses were given if the MAP was $<80 \%$ of baseline MAP or the systolic blood pressure (SBP) was $<90 \mathrm{mmHg}$. If the heart rate was $>70 \mathrm{bpm}$, phenylephrine $(50-100 \mu \mathrm{g})$ was administered, and if the heart rate was $<70 \mathrm{bpm}$, ephedrine $(5-10 \mathrm{mg}$ ) was administered. The unit also allows for a fixed-rate, prophylactic phenylephrine infusion $(25 \mu \mathrm{g} / \mathrm{min})$ to be employed at the attending anaesthetist's discretion. A $1 \mathrm{~L}$ co-load of Ringer's lactate was administered rapidly via an $18 \mathrm{G}$ intravenous line after insertion of the spinal anaesthetic. After delivery, 3 IU oxytocin was administered as a slow injection and 7 IU oxytocin was given in the subsequent litre of fluid as an infusion. Postoperatively all patients received 20 IU oxytocin in 1 L Ringer's lactate over 8 hours, as per national protocol.

All haemodynamic data were recorded with Nihon-Kohden Lifescope monitors (BSM 3562, Nihon-Koden, Japan). Data were manually recorded by the attending anaesthetist, entered into a Microsoft Excel 2013 (Microsoft, USA) spreadsheet by a research assistant, and then checked by one of the study investigators to ensure data fidelity. Data were exported to a statistical program (Stata 13, StataCorp, USA) for further analysis.

\section{Outcome}

The primary outcome was the prediction of hypotension, defined as an SBP $<90 \mathrm{mmHg}$, from the time of spinal insertion until 15 minutes after delivery of the baby.

\section{Statistical analysis}

We planned to examine six candidate variables (body mass index (BMI), baseline heart rate, baseline MAP, maternal age, urgency of surgery (elective v. non-elective) and preoperative haemoglobin) as predictors of hypotension, and used a projected incidence of $30 \%$ hypotension. To avoid model over-fitting, it is suggested to have at least $10-15$ events per variable tested in a regression model. ${ }^{[6,7]} \mathrm{We}$ therefore required 60 events and recruitment of at least 180 patients. We aimed to recruit a minimum of 440 patients.
Baseline characteristics of included patients were reported as means (standard deviation) for continuous normally distributed variables, and as numerical counts and percentages for categorical variables. Comparisons between normally distributed continuous data were done using Student's $t$-test, and categorical data were analysed using the $\chi^{2}$ test. The Shapiro-Wilk test was used for testing whether data were normally distributed.

We performed backward stepwise logistic regression with bootstrapping (exclusion threshold $p>0.1$ ) on the candidate variables to determine their association with the primary outcome. We assessed collinearity using the variance inflation factor. Variables with a variance inflation factor $>10$ were considered to be collinear, and we then excluded these variables from the analysis. Empirical cut-point estimations were determined on the remaining significant variables $(p<0.05)$ using the method described by Liu. ${ }^{[8]}$

We developed a simplified risk score by assigning one point to variables with values greater than the cut-point (heart rate, age) or less than the cut-point (MAP). We then determined model discrimination ( $c$-statistic) and calibration. We reported the odds ratios, $95 \%$ confidence intervals (CIs) and associated $p$-values to 3 decimal places, with $p$-values less than 0.001 reported as $p<0.001$. For all tests, we used an alpha $<0.05$ level of significance.

The Strengthening the Reporting of Observational Studies in Epidemiology (STROBE) guideline for the reporting of observational studies was followed. ${ }^{[9]}$

\section{Results}

\section{Eligibility}

The patient flow diagram (Fig. 1) shows that a total of 522 patients were initially included in the study. Eighteen were excluded: 2 had a general anaesthetic as the primary anaesthetic, 3 received the incorrect bupivacaine dose, and 13 required conversion to general anaesthesia during the time period assessed (spinal anaesthesia failed in 11 of these patients, one had postpartum haemorrhage, and one had an iatrogenic bowel injury). The final analysis included 504 patients.

The patient characteristics are outlined in Table 1.

The primary outcome was defined as an SBP of $<90 \mathrm{mmHg}$ from the time of spinal insertion until 15 minutes after delivery of the baby. We performed backward stepwise logistic regression using the candidate variables BMI, baseline heart rate, baseline MAP, maternal age, urgency of surgery (elective v. non-elective), and preoperative haemoglobin to determine their association with the primary outcome. The results of the backward stepwise logistic regression analysis with bootstrapping are shown in Table 2.

This analysis confirmed that advancing age, a higher preoperative heart rate and a lower preoperative MAP were associated with an

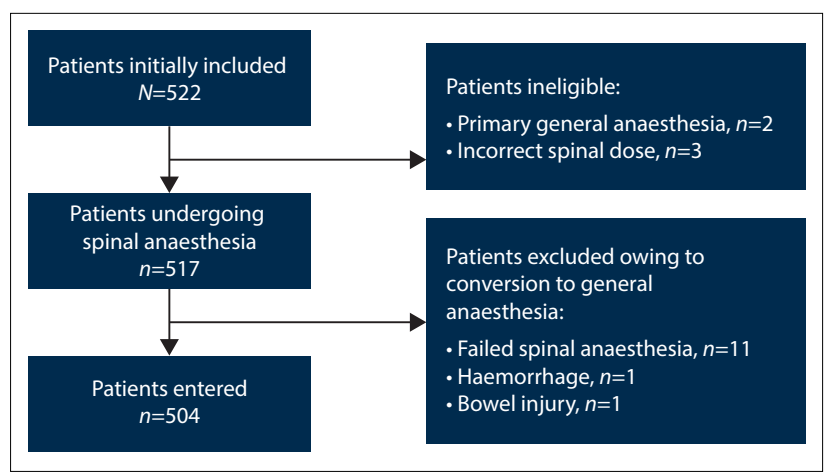

Fig. 1. Patient flow chart. 
Table 1. Characteristics of recruited patients, comparing patients with an SBP of $\geq 90 \mathrm{mmHg}$ during the time period assessed with those with an SBP of $<90 \mathbf{~ m m H g}$

\begin{tabular}{|c|c|c|c|c|}
\hline Characteristics & $\begin{array}{l}\text { All patients } \\
(N=504)\end{array}$ & $\begin{array}{l}\text { Normotensive group } \\
(\mathrm{SBP} \geq 90 \mathrm{mmHg}) \\
(\mathrm{N}=351)\end{array}$ & $\begin{array}{l}\text { Hypotensive group } \\
(\mathrm{SBP}<90 \mathrm{mmHg}) \\
(N=153)\end{array}$ & $p$-value \\
\hline Age (yr), mean (SD) & $27.4(6.3)$ & $26.9(6.5)$ & $28.5(5.4)$ & $0.009^{*}$ \\
\hline Weight $(\mathrm{kg})$, mean $(\mathrm{SD})$ & $81(16.8)$ & $81(15.8)$ & $83(18.8)$ & 0.080 \\
\hline Height $(\mathrm{cm})$, mean $(\mathrm{SD})$ & $158(7.5)$ & $158(7.6)$ & $158(7.2)$ & 0.673 \\
\hline BMI $\left(\mathrm{kg} / \mathrm{m}^{2}\right)$, mean $(\mathrm{SD})$ & $32.6(6.8)$ & $32.2(6.4)$ & $33.4(7.5)$ & 0.063 \\
\hline Haemoglobin (g/dL), mean (SD) & $11.4(1.6)$ & $11.3(1.7)$ & $11.5(1.6)$ & 0.215 \\
\hline Hypertensive disease, $n(\%)$ & $71(14.1)$ & $61(17.4)$ & $10(6.5)$ & $<0.001^{*}$ \\
\hline Non-elective category, $n(\%)$ & $154(30.6)$ & $120(34.2)$ & $34(22.2)$ & $0.007^{\star}$ \\
\hline Baseline heart rate (bpm), mean (SD) & $92(15.0)$ & $91(15.0)$ & $94(14.7)$ & 0.088 \\
\hline Baseline MAP (mmHg), mean (SD) & $97(14.2)$ & $99(14.2)$ & $93(13.6)$ & $<0.001^{*}$ \\
\hline Baseline SBP (mmHg), mean (SD) & $128(16.8)$ & $131(16.9)$ & $122(14.8)$ & $<0.001^{*}$ \\
\hline Total intraoperative fluid (mL), mean (SD) & $1385(542)$ & $1302(495)$ & $1579(598)$ & $<0.001^{*}$ \\
\hline Intraoperative blood loss $(\mathrm{mL})$, mean $(\mathrm{SD})$ & $577(186)$ & $565(177)$ & $607(203)$ & $0.019^{*}$ \\
\hline
\end{tabular}

Table 2. Results of backward stepwise logistic regression analysis of all significant candidate predictors for SBP $<90$ mmHg during the study period

\begin{tabular}{lccl}
\hline Variable & OR & $\mathbf{9 5 \%}$ CI & $p$-value \\
\hline Maternal age & 1.05 & $1.02-1.08$ & $0.002^{*}$ \\
Preoperative MAP & 0.97 & $0.95-0.98$ & $<0.001^{*}$ \\
Preoperative heart rate & 1.02 & $1.00-1.03$ & 0.012 \\
$\begin{array}{l}\text { SBP = systolic blood pressure; OR = odds ratio; } \mathrm{CI}=\text { confidence interval; } \mathrm{MAP}=\text { mean } \\
\text { arterial pressure. }\end{array}$ \\
`Statistically significant $(p<0.05)$.
\end{tabular}

increased incidence of hypotension following spinal anaesthesia. There was no significant collinearity between these variables. The empirical cut-point yielded cut-point risk thresholds of maternal age $>25$ years (age $>25=1$ ), preoperative heart rate $>90 \mathrm{bpm}$ (heart rate $>90=1)$ and preoperative MAP $<90 \mathrm{mmHg}(\mathrm{MAP}<90=1)$. We used these values to generate a pulse rate, age and MAP score (the PRAM score). The incidence of hypotension in each of the risk categories is shown in Table 3.

The overall incidence of hypotension in the patient group was $30.4 \%$. As shown in Fig. 2, the $c$-statistic for the prediction of an SBP $<90 \mathrm{mmHg}$ was 0.63 (95\% CI 0.58 - 0.66).

The model showed good calibration (Fig. 3). Calibration was assessed graphically by plotting the observed outcome against the predicted probability of SBP $<90 \mathrm{mmHg}$.

\section{Discussion}

This prospective, observational single-centre study aimed to identify preoperative risk factors for obstetric spinal hypotension and then to develop a preliminary preoperative risk scoring system for the development of intraoperative hypotension. We found that higher preoperative heart rate, lower preoperative MAP and advancing maternal age were associated with an increased incidence of hypotension ( $\mathrm{SBP}<90 \mathrm{mmHg}$ ). We then developed a preliminary scoring system (the PRAM score) to identify patients at increased risk.

Obstetric spinal hypotension is common, with up to $80 \%$ of cases requiring treatment with a vasopressor. ${ }^{[10]}$ It is potentially life threatening and is associated with nausea and vomiting, ${ }^{[2]}$ loss of consciousness, cardiac arrest, death and fetal compromise. ${ }^{[3]}$ Spinal hypotension is linked to more than half of the anaesthetic
Table 3. Incidence of hypotension in each of the PRAM score risk categories

\begin{tabular}{|c|c|c|c|}
\hline PRAM score & $\begin{array}{l}\text { Normotension } \\
(N=351)(\mathrm{SBP} \\
\geq 90 \mathrm{mmHg}) \\
\%(n)\end{array}$ & $\begin{array}{l}\text { Hypotension } \\
(N=153) \text { (SBP } \\
<90 \mathrm{mmHg}) \\
\%(n)\end{array}$ & Total, $N$ \\
\hline 0 & $81.1(43)$ & $18.9(10)$ & 53 \\
\hline 1 & 78.4 (149) & $21.6(41)$ & 190 \\
\hline 2 & $64.2(136)$ & $35.8(76)$ & 212 \\
\hline 3 & $46.9(23)$ & $53.1(26)$ & 49 \\
\hline Total & $69.6(351)$ & $30.4(153)$ & 504 \\
\hline
\end{tabular}

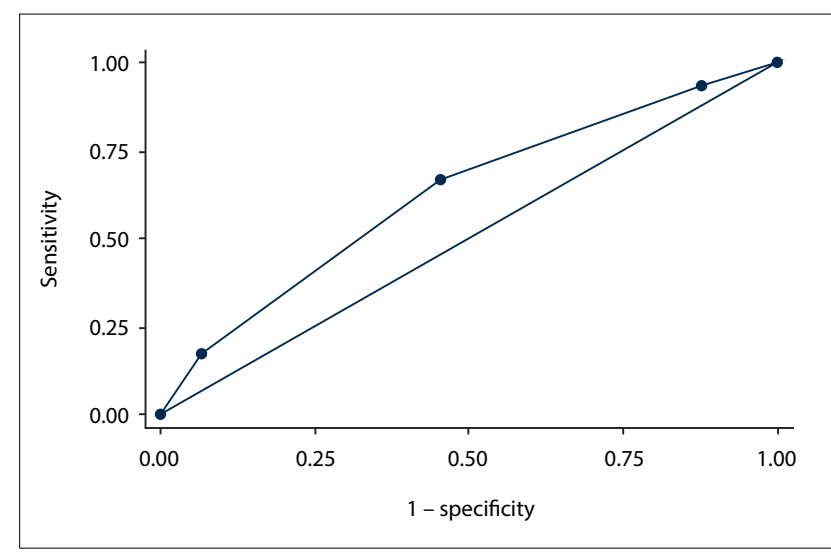

Fig. 2. Receiver operator curve for PRAM score predicting $S B P<90 \mathrm{mmHg}$ from the time of spinal insertion until 15 minutes after delivery of the baby. Area under the curve $=0.626$. (PRAM = pulse rate, age and mean arterial pressure; $S B P=$ systolic blood pressure.)

deaths in SA. ${ }^{[4]}$ Routine risk profiling for the development of spinal hypotension holds many attractions, especially in the resourceconstrained environment. Anaesthetists in developing-world conditions face unique challenges, including a lack of training and experience. ${ }^{[11]}$ There is an urgent need to better identify patients at risk and implement strategies to prevent associated morbidity and mortality. Scoring systems that identify patients at risk could 


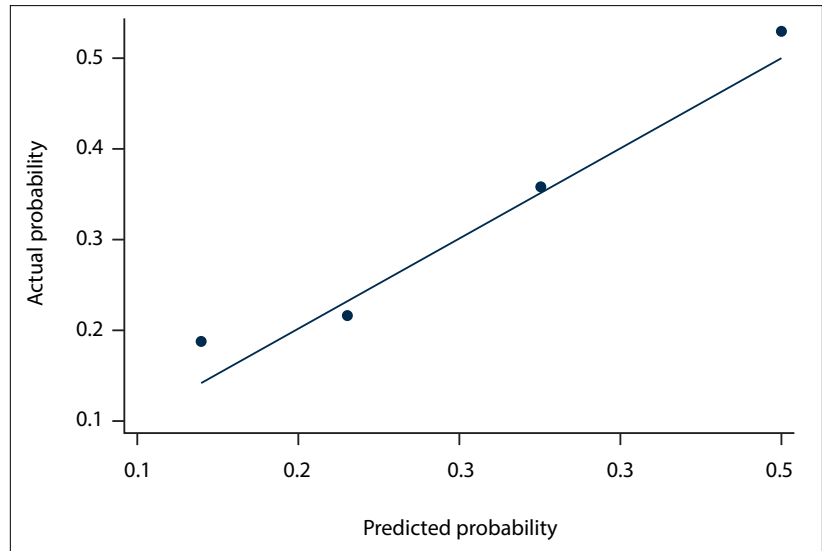

Fig. 3. Calibration of PRAM score for the prediction of $S B P<90 \mathrm{mmHg}$ from the time of spinal insertion until 15 minutes after delivery of the baby: a plot of expected (solid line) v. actual risk (dots) of spinal hypotension (SBP $<90 \mathrm{mmHg}) .(P R A M=$ pulse rate, age and mean arterial pressure; $S B P=$ systolic blood pressure.)

potentially enhance anaesthetic planning, guide clinical decisionmaking and lead to timeous referral where appropriate. In addition, routine scoring would sensitise clinicians to this important clinical problem.

The incidence of hypotension is difficult to quantify owing to inconsistencies in the definitions used across studies. Klohr et al. ${ }^{[1]}$ found 15 different definitions across 63 different publications, with a 10 -fold difference in incidence, depending on the definition used. We used an absolute cut-off of SBP $<90 \mathrm{mmHg}$ to define hypotension, as this is both clinically relevant and simple to use.

In choosing the candidate variables for this study, a higher priority was given to parameters that are easily measured and readily available and would have practical significance to the attending anaesthetist in a resource-constrained setting. Four of the candidate variables in this study have previously shown promise as predictors of spinal hypotension. Baseline heart rate $\mathrm{e}^{[12]}$ and advancing maternal age $\mathrm{e}^{[13,14]}$ were predictors in retrospective studies, which our study confirmed. A retrospective study demonstrated an association between a higher preoperative MAP and significant hypotension under spinal anaesthesia in elective cases. ${ }^{[13]}$ However, these authors used a relative definition for hypotension of an MAP reduction of $>30 \%$ from baseline rather than an absolute definition, which may explain why our study found the opposite. BMI has previously been shown to predict hypotension in obstetric spinal anaesthesia, ${ }^{[14,15]}$ but our study did not confirm this association. We also included urgency of surgery and baseline haemoglobin as variables for theoretical reasons, despite a lack of published evidence. Neither was shown to be predictive of spinal hypotension. We did not aim to include methods of prediction such as heart rate variability ${ }^{[1,17]}$ or the perfusion index, ${ }^{[18]}$ because the equipment required is not universally available.

There are no simple scoring systems in use for the prediction of obstetric spinal hypotension. Attempts to use computer-based predictive models, such as artificial neural networks in the prediction of general spinal hypotension, ${ }^{[19]}$ are restricted by their complexity in resource-constrained settings. Practical scoring systems such as the shock index are already being used in postpartum haemorrhage to establish 'alert thresholds' for use in resource-constrained environments. ${ }^{[20]}$ Similar systems in obstetric anaesthesia have significant appeal in low-resource environments. ${ }^{[21]}$ Based on our findings, we developed a simple scoring system designed to identify the high-risk patient for spinal hypotension. We used cut-point estimates to choose clinically relevant criteria and demonstrated that high-risk patients for hypotension can be identified preoperatively.

The incidence of hypotension ( $\mathrm{SBP}<90 \mathrm{mmHg}$ ) in our cohort was $30.4 \%$. Given this high event rate, it could be argued that there is little practical value in attempting to predict hypotension. However, patients with a PRAM score of 3 were more than twice as likely to experience hypotension as those with a score of $0-1(53.8 \% \mathrm{v}$. $21.6 \%)$. Preoperative identification of high-risk patients could enable targeted prophylactic interventions such as use of a prophylactic phenylephrine infusion. It could also enable timeous referral to a specialist centre if expertise is lacking. In addition, the PRAM score would sensitise clinicians to an important outcome in maternal anaesthesia.

\section{Study limitations}

There are several limitations to this study. We used a single blood pressure reading for a baseline measurement: this potentially overestimates blood pressure, owing to maternal anxiety. The absolute definition of hypotension minimises the impact of this problem, but does mean that hypertensive patients who have a greater reduction in baseline blood pressure may be excluded. This problem highlights the need for a consensus definition for obstetric spinal hypotension. Although the cohort consisted of 504 patients, using prospectively collected data, much larger numbers are required to generate a more robust scoring system. While the numbers in our trial are comparable with other studies in the field, ${ }^{[12-15]}$ in-hospital mortality scores such as the Preoperative Score to Predict Postoperative Mortality (POSPOM) were derived from over five and a half million patients. ${ }^{[22]}$ We also used a single centre for data collection: differences in patient populations and anaesthetic techniques can be expected to alter both the incidence and the mechanism of hypotension. We have not validated our model: prospective, multicentre external validation will therefore be required to ensure reproducibility and reduce the fragility of results. However, the PRAM score shows promise as a simple tool to quickly evaluate perioperative risk in the obstetric group.

\section{Conclusions}

This study found preoperative heart rate, preoperative MAP and advancing maternal age to be predictive of hypotension. The PRAM score shows promise as a preliminary method to identify the highrisk patient for obstetric spinal hypotension using basic, readily available clinical parameters. Further prospective, multicentre studies are required to confirm our findings.

\section{Acknowledgements. None.}

Author contributions. DGB contributed to conceptualisation, design, analysis and interpretation of data, drafted the manuscript, and approved the final version for publication: CC contributed to conceptualisation and design, critical revision of important scientific content, and approved the final version for publication; MG contributed to conceptualisation and design, critical revision of important scientific content, and approved the final version for publication; and RNR contributed to conceptualisation, design, analysis and interpretation of data, drafted the manuscript, and approved the final version for publication.

Funding. None.

Conflicts of interest. None.

1. Klohr S, Roth R, Hofmann T, Rossaint R, Heesen M. Definitions of hypotension after spinal anaesthesia for caesarean section: Literature search and application to parturients. Acta Anaesthesiol Scand 2010;56(7):909-921. https://doi.org/10.1111/j.1399-6576.2010.02239.x 
2. Ngan Kee WD, Khaw KS, Lee BB, Lau TK, Gin T. A dose-response study of prophylactic intravenous ephedrine for the prevention of hypotension during spinal anesthesia for cesarean delivery. Anesth ephedrine for the prevention of hypotension during spinal anesthesia for cesare
Analg 2000;90(6):1390-1395. https://doi.org/10.1097/00000539-200006000-00024

3. Reynolds F, Seed PT. Anaesthesia for caesarean section and neonatal acid-base status: A meta-analysis. Reynolds F, Seed PT. Anaesthesia for caesarean section and neonatal acid-base status
Anaesthesia 2005;60(7):636-653. https://doi.org/10.1111/j.1365-2044.2005.04223.x

4. Pattinson RC, ed. Saving Mothers 2011-2013: The Sixth Report of the National Committee for Confidential Enquiries into Maternal Deaths in South Africa. Pretoria: Government Printer, 2014

5. Bishop DG. Predicting spinal hypotension during caesarean section. South Afr J Anaesth Analg 2014;20(4):170-173. https://doi.org/10.1080/22201181.2015.959336

6. Peduzzi P, Concato J, Kemper E, Holford TR, Feinstein AR. A simulation study of the number of events per variable in logistic regression analysis. J Clin Epidemiol 1996;49(12):1373-1379. https://doi. org $/ 10.1016 / 50895-4356(96) 00236-3$

7. Steyerberg EW, Eijkemans MJ, Habbema JD. Stepwise selection in small data sets: A simulation study of bias in logistic regression analysis. J Clin Epidemiol 1999;52(10):935-942. https://doi,org/10.1016/ S0895-4356(99)00103-1

8. Liu X. Classification accuracy and cut point selection. Stat Med 2012;31(23):2676-2686. https://doi. org/10.1002/sim.4509

9. Vandenbroucke JP, von Elm E, Altman DG, et al. Strengthening the Reporting of Observational Studies in Epidemiology (STROBE): Explanation and elaboration. Ann Intern Med 2007;147(8):573-577. https://doi.org/10.7326/0003-4819-147-8-200710160-00010

10. Rout CC, Rocke DA. Prevention of hypotension following spinal-anesthesia for cesarean-section. Int Anesthesiol Clin 1994;32(2):117-135.

11. Reed A, Mumba JM, Dyer R. A spotlight on obstetric anesthesia in the developing world: Finally getting the attention it deserves. Anesth Analg 2015;120(6):1179-1181. https://doi.org/10.1213/ ANE.000000000000072

12. Frölich MA, Caton D. Baseline heart rate may predict hypotension after spinal anesthesia in prehydrated obstetrical patients. Can J Anaesth 2002;49(2):185-189. https://doi.org/10.1007/BF03020493

13. Maayan-Metzger A, Schushan-Eisen I, Todris L, Etchin A, Kuint J. Maternal hypotension during elective cesarean section and short-term neonatal outcome. Am J Obstet Gynecol 2010;202(1):56.el56.e5. https://doi.org/10.1016/j.ajog.2009.07.012
14. Brenck F, Hartmann B, Katzer C, et al. Hypotension after spinal anesthesia for cesarean section: Identification of risk factors using an anesthesia information management system. J Clin Monit Identification of risk factors using an anesthesia information managen
Comput 2009;23(2):85-92. https://doi.org/10.1007/s10877-009-9168-x

15. Nani FS, Torres MLA. Correlation between the body mass index (BMI) of pregnant women and the development of hypotension after spinal anesthesia for cesarean section. Braz J Anesthesiol 2011;61(1):21-30. https://doi.org/10.1016/S0034-7094(11)70003-4

16. Hanss R, Bein B, Ledowski T, et al. Heart rate variability predicts severe hypotension after spinal anaesthesia for elective cesarean delivery. Anesthesiology 2005;102(6):1086-1093.

17. Bishop DG, Cairns C, Grobbelaar M, Rodseth RN. Heart rate variability as a predictor of hypotension following spinal for elective caesarean section: A prospective observational study. Anaesthesia 2017;72(5):603-608. https://doi.org/10.1111/anae.13813

18. Toyama S, Kakumoto M, Morioka M, et al. Perfusion index derived from a pulse oximeter can predict the incidence of hypotension during spinal anaesthesia for caesarean delivery. Br J Anaesth 2013;111(2):235-241. https://doi.org/10.1093/bja/aet058

19. Lin CS, Chiu JS, Hsieh MH, Mok MS, Li YC, Chiu HW. Predicting hypotensive episodes during spinal anesthesia with the application of artificial neural networks. Comput Methods Programs Biomed 2008;92(2):193-197. https://doi.org/10.1016/j.cmpb.2008.06.013

20. Nathan HL, El Ayadi A, Hezelgrave NL, et al. Shock index: An effective predictor of outcome in Nathan HL, El Ayadi A, Hezelgrave NL, et al. Shock index: An effective predictor of outcome
postpartum haemorrhage? BJOG 2015;122(2):268-275. https://doi.org/10.1111/1471-0528.13206

21. Bishop DG, Rodseth RN, Dyer RA. Recipes for obstetric spinal hypotension: The clinical context Bishop DG, Rodseth RN, Dyer RA. Recipes for obstetric spinal hypotension: The clinical
counts. S Afr Med J 2016;106(9):861-864. https://doi.org/10.7196/SAMJ.2016.v106i9.10877

22. Le Manach Y, Collins G, Rodseth R, et al. Preoperative Score to Predict Postoperative Mortality (POSPOM): Derivation and validation. Anesthesiology 2016;124(3):570-579. https://doi.org/10.1097 ALN.0000000000000972

Accepted 27 July 2017. 